This document was prepared in conjunction with work accomplished under Contract No. DE-AC09-96SR18500 with the U.S. Department of Energy.

This work was prepared under an agreement with and funded by the U.S. Government. Neither the U. S. Government or its employees, nor any of its contractors, subcontractors or their employees, makes any express or implied: 1 . warranty or assumes any legal liability for the accuracy, completeness, or for the use or results of such use of any information, product, or process disclosed; or 2 . representation that such use or results of such use would not infringe privately owned rights; or 3 . endorsement or recommendation of any specifically identified commercial product, process, or service. Any views and opinions of authors expressed in this work do not necessarily state or reflect those of the United States Government, or its contractors, or subcontractors. 


\title{
Neutron Activation Analysis Applications at the Savannah River Site Using an Isotopic Neutron Source
}

\author{
D.P. DiPrete, C. C. DiPrete, and R.A. Sigg \\ Savannah River National Laboratory \\ Washington Savannah River Company \\ Aiken, SC 29808 \\ david.diprete@srnl.doe.gov
}

\begin{abstract}
NAA using ${ }^{252} \mathrm{Cf}$ is used to address important areas of applied interest at SRS.

Sensitivity needs for many of the applications are not severe; analyses are

accomplished using a $21 \mathrm{mg}{ }^{252} \mathrm{Cf}$ NAA facility. Because NAA allows analysis of bulk samples, it offers strong advantages for samples in difficult-to-digest matrices when its sensitivity is sufficient. Following radiochemical separation with stable carrier addition, chemical yields for a number methods are determined by neutron activation of the stable carrier. In some of the cases where no suitable stable carriers exist, the source has been used to generate radioactive tracers to yield separations.
\end{abstract}

\section{INTRODUCTION}


The Savannah River National Laboratory (SRNL) has used a ${ }^{252} \mathrm{Cf}$ neutronactivation-analysis (NAA) facility ${ }^{1}$ routinely since 1976 . Methods for a much larger and more highly automated activation analysis system at the Savannah River Site (SRS) C-Reactor were developed at the ${ }^{252} \mathrm{Cf}$ facility to support the national uranium resource evaluation (NURE) program. In its heyday in the late 1970s and early 1980s, the reactor facility provided fully-automated, resource-driven activation, counting, sample storage and retrieval for over 70,000 soil and sediment samples per year. Uranium was determined by delayed neutron counting, and a broad suite of other activated elements were measured by gamma-ray spectrometry.

Although the C-Reactor facility is no longer in use, the SRNL ${ }^{252} \mathrm{Cf}$ facility continues to provide multi-element analyses on solid and liquid samples. The isotopic neutron source supports applied research programs at SRNL as well as other SRS programs for environmental and waste management studies. This paper describes the facility and several specific applications.

\section{EXPERIMENTAL}

The present SRNL NAA facility (Fig. 1) holds eight doubly encapsulated ${ }^{252} \mathrm{Cf}$ pods in a zircalloy source holder near the bottom of a 4-meter deep, 1.2-meter diameter tank. The tank contains deionized light water, and the source holder is in the annulus of a second tank filled with $\mathrm{D}_{2} \mathrm{O}$. The heavy water tank has a total of eighteen irradiation ports that are arranged in two rings ( $\sim 5.8$ and $\sim 11.2 \mathrm{~cm}$ radii) concentric to the source holder. Three of the positions originally could be pneumatically fed, although the pneumatic system is currently out of service. Samples are currently 
introduced manually at 8 functioning locations. All irradiation positions were designed to accept 13-mL high-density-polyethylene containers ("rabbits"). At one time, the facility contained eight sources having a total $150-\mathrm{mg}$ mass of ${ }^{252} \mathrm{Cf}$. Its present ${ }^{252} \mathrm{Cf}$ content of about 21 milligrams gives a thermal-neutron flux of about $1 \times 10^{7} \mathrm{n} /\left(\mathrm{cm}^{2} \mathrm{sec}\right)$ at the highest flux irradiation positions. The heavy water tank minimizes flux gradients across samples and it gives a flux that is only a factor of three lower at the outer irradiation ring. Although the flux is low relative to reactor facilities, it continues to offer sufficiently sensitive analyses for many on-site applied research efforts and other SRS programs. Roughly 30 elements can be detected at low- and sub-part-per-million levels under routine irradiation and counting conditions.

Germanium detectors for gamma-ray spectrometry are located in a counting vault that is separated from the room housing the NAA tank by a 1.2-meter-thick concrete wall. A pneumatic transfer system allowing analysis of elements having short-lived activation products, and automated cyclic irradiations could previously be performed to enhance sensitivity for short-lived activation and fission products. Samples containing small quantities of fissile materials were also analyzed using this cyclic capability via delayed neutron counting using ${ }^{3} \mathrm{He}$-filled proportional counters. Efforts are currently underway to restore this capability.

Over the years, gamma-ray spectrometry at the facility passed through several generations of computers, software, multichannel analyzers, and germanium detectors. Available germanium detectors presently include a conventional $30 \% \mathrm{p}$ - 
type coaxial detector, a $40 \%$ n-type coaxial detector, a compton suppressed Canberra telescope detector, and 4 large-area-low-energy-photon spectrometers. For activation products having suitable half-lives, a 50\% n-type and a $40 \%$ p-type coaxial both enclosed in Changer Labs automated sample changers are also available for more cost-effective counting.

A 3 X3 NaI well detector is available adjacent to the ${ }^{252} \mathrm{Cf}$ well for applications analyzing for short $(<30$ second) half life isotopes where the resolution of the $\mathrm{NaI}$ is adequate. Efforts are underway to procure a $300 \mathrm{cc}$ HPGe well detector contained in a third Changer labs automated sample changer. Conventional nuclear instrument module (NIM) amplifiers and analog to digital converters (ADC) comprise most of the data acquisition hardware, but a Canberra digital signal processing (DSP) system is brought to bear for high-count-rate applications. All of the data acquisition systems take advantage of pulse-pile-up rejection and dead-time corrections that are available using present-day commercially available electronics. The DSP electronics offer a clear count-rate-throughput advantage relative to conventional amplifiers and ADCs when count rates are high.

Data acquisition devices are interfaced to Intel-based personal computers through Canberra Industries (CI) acquisition interface modules. CI's Genie2000 software controls data acquisition, analyzes gamma-ray spectra and simplifies quality assurance. Originally, SRNL applied absolute methods to interpreting counting data; these relied on knowledge of detector efficiencies, neutron energy spectra, neutron cross-sections, etc. to determine elemental contents. Most NAA applications now 
rely on direct comparisons of results on "unknowns" to those determined on standards.

\section{RESULTS AND DISCUSSION}

Applications of NAA at the SRNL have included elemental analysis on a wide array of SRS Site sample matrices, both radioactive and non-radioactive. In addition to direct bulk-sample NAA, numerous radiochemical analyses rely on the facility for production of short-lived tracers, yielding by activation of carriers and small-scale isotope production for separation methods testing.

Other multielement analysis methods, particularly inductively coupled plasma atomic emission (ICP-AE) and inductively coupled plasma mass spectrometry (ICP-MS), now provide sensitivities on dissolved samples that are often better than those available by NAA using low-flux isotopic sources. Despite the low neutron flux, the NAA facility's sensitivity is adequate for many analytical projects, and it is a more cost-effective choice in many cases because it allows analysis of bulk samples without chemical digestion. The source is heavily relied on for bulk chlorine analyses to determine whether items purchased by SRS that will come in contact with stainless steel meet the Site chloride level specifications.

NAA sensitivity is dependent on neutron flux, irradiation time, sample mass, detector counting efficiency and background, and count time. Figure $2 \mathrm{a}, 2 \mathrm{~b}$, and $2 \mathrm{c}$ shows sensitivities $^{2}$ for NAA at the SRNL NAA facility using routine activation and counting protocols; the figure assumes that activation and counting times individually are the lesser of two half lives or two days. Most routine analyses, however, receive 
relatively short exposures and counts while meeting analytical goals. Since counting backgrounds for NAA are matrix dependent due to activation of other species in the matrix, the sensitivities shown are for bounding-case matrices that offer few if any interferences.

Chlorine analyses have been a mainstay for the facility. These data are used to assure that chlorine concentrations in materials that may come in contact with stainless steels meet technical specifications. On solutions, ion chromatography methods are more sensitive for chlorine than SRNL's NAA facility. However, in solid matrices, NAA becomes competitive with wet chemistry methods when the requirements for digestion require large dilution factors. The NAA facility as it currently stands can easily meet the Savannah River Site's total chlorine concentration limit of $250 \mathrm{ppm}$ on such matrices as gasket materials, labels, certain metals and alloys, etc. For a typical 30-minute irradiation, 5-minute decay period and 16.6-minute count (Fig. 2b), the chlorine sensitivity is about $14 \mathrm{ppm}$ on a 5 gram sample. If appreciable levels of elements (such as sodium) were present, they would degrade sensitivity by significantly raising the Compton background below ${ }^{38} \mathrm{Cl}$ 's 1643 and $2168 \mathrm{keV}$ gamma-ray peaks.

Although in less demand than chlorine analyses, analyses for iodine, bromine, sodium, and silver are carried out periodically.

Radiochemical separations are required for analysis of beta emitters that have no significant gamma-ray emission intensity. The matrices and radionuclide distributions of samples analyzed at SRNL vary widely. Do to the non-homogenous 
nature of the sample load, the separations generally require yielding by independent analytical methods to account for losses of analyte in separation processes. Yields may be determined by comparing the amount of carrier or radioactive tracer that is recovered after separation to the known amount added to the sample before commencing the separation. Specific examples of using neutron activation for tracer preparation and for carrier measurements are given in discussions that follow. Although other instrumentation (AA, ICP-AES, ICP-MS, etc...) are available to support carrier measurements, NAA via the ${ }^{252} \mathrm{Cf}$ facility can offer some significant advantages. The facility currently supports carrier analyses for ${ }^{90} \mathrm{Sr},{ }^{129} \mathrm{I},{ }^{79} \mathrm{Se}$, and ${ }^{151} \mathrm{Sm}$. Separations have also been traced with short lived isotopes generated by the source, namely ${ }^{99 \mathrm{~m}} \mathrm{Tc}-99 \mathrm{~m}$ and ${ }^{149} \mathrm{Pm}$.

Analyses for ${ }^{90} \mathrm{Sr}$, a pure beta emitting fission product having a $30-\mathrm{yr}$ half life, are required to support development of high-level waste vitrification methods, and other samples of interest to waste management and environmental restoration studies. In early efforts, ${ }^{85} \mathrm{Sr}$ tracer measurement data provided yields for ${ }^{90} \mathrm{Sr}$ analyses; however, the presence of ${ }^{85} \mathrm{Sr}$ interferes with beta activity measurements of ${ }^{90} \mathrm{Sr} /{ }^{90} \mathrm{Y}$ by liquid scintillation (LSC) and Cerenkov counting. This interference and its resulting sensitivity degradation are avoided by using strontium carrier for yielding. After LSC counting is completed, the same small 7-mL vials used for LSC counting are sealed in NAA rabbits, activated and counted. In this way, yielding is conveniently and cost effectively accomplished by NAA without returning the sample to a radiologically posted laboratory, without reopening the sample and with minimal additional 
preparation. The SRNL radiochemistry laboratory uses extraction chromatography columns (Eichrom Industries) for strontium separations. Using 5-mg strontium carrier, an amount recommended by Eichrom to avoid overloading the column, NAA provides accurate yields by measuring the $388 \mathrm{keV}$ gamma rays of the ${ }^{87 \mathrm{~m}} \mathrm{Sr}$ product.

${ }^{129} \mathrm{I}$ is a fission product with a half-life of about 16 million years. It is very mobile in the environment due to its low retention in sub-surface soil. ${ }^{129} \mathrm{I}$ has long been considered a potentially problematic radionuclide in low-level radioactive waste disposal ${ }^{4}$. As a result considerable emphasis has been placed on ${ }^{129} \mathrm{I}$ analyses on the wide variety of radioactive waste streams generated at the Savannah River Site. The neutron flux at the SRNL NAA facility is too low to determine ${ }^{129}$ I at typical environmental levels by activation to ${ }^{130} \mathrm{I}$; however, the facility is well suited to support carrier recovery measurements of ${ }^{129} \mathrm{I}$ radiochemical analyses. Currently SRNL uses a variant of the silver iodide precipitation method prescribed for EPA drinking water analyses ${ }^{5}$. As the sample matrix varies widely for the various waste streams, the potential for interferences using a gravimetric-based chemical yielding approach is substantial. Once the AgI mounts are analyzed by low energy gamma ray spectrometry for the $\mathrm{x}$-rays and $37 \mathrm{keV}$ gamma ray generated from ${ }^{129} \mathrm{I}$ decay, the mount is then activated to measure the ${ }^{127}$ I carrier concentration to yield the separation.

${ }^{79} \mathrm{Se}$ is a soft beta emitting fission product which has a long (2.9E5 year) half-life. Although low on the actinides fission yield curves, because of the long half life the 
isotope has become one of the isotopes of concern for the long term storage radioactive waste facilities. Radiochemical separations for the isotope are carried out at SRNL by precipitating ${ }^{79} \mathrm{Se}$ as the metal following the addition of selenium carrier. ${ }^{79} \mathrm{Se}$ is then further purified with a solvent-solvent tri-butyl phosphate based extraction. ${ }^{79}$ Se concentrations are determined off the purified extracts using liquid scintillation analyses. Stable selenium concentrations are rapidly determined by activating ${ }^{76} \mathrm{Se}$ to ${ }^{76 \mathrm{~m}} \mathrm{Se}\left(\mathrm{t}_{1 / 2}=17.4 \mathrm{~s}\right)$. The $162 \mathrm{keV}$ gamma ray is measured with a $\mathrm{NaI}$ well detector located adjacent to the ${ }^{252} \mathrm{Cf}$ well for the purposes of analyzing short lived activation products.

Site programs also require analyses of ${ }^{99} \mathrm{Tc}$, another long-lived $\left(2 \times 10^{5} \mathrm{yr}\right)$, low-energy beta-emitting fission product. These analyses play a significant role in developing and testing flow sheets for high level waste vitrification processes. The measurements also support other waste management and environmental programs. There are no stable technetium isotopes for yielding ${ }^{99} \mathrm{Tc}$. One analysis path for ${ }^{99} \mathrm{Tc}$ includes ${ }^{99 \mathrm{~m}} \mathrm{Tc}$ tracer addition for chemical yielding, radiochemical separation by extraction chromatography, tracer activity measurement by gamma-ray spectrometry and ${ }^{99} \mathrm{Tc}$ activity determination by liquid scintillation counting Preparation of ${ }^{99 \mathrm{~m}} \mathrm{Tc}$ by neutron activation of ammonium molybdate is the practical choice for SRNL to generate tracer, the ${ }^{99} \mathrm{Tc}$ protocols used at SRNL are discussed in detail in reference 6.

${ }^{151} \mathrm{Sm}$ and ${ }^{147} \mathrm{Pm}$ are the pure beta emitters among the lanthanide fission products that require characterization by radiochemical separations. Routinely, lanthanide 
extractions based on Eichrom's Ln resin are conducted and are traced with stable samarium carrier. Aliquots of separated $\mathrm{Pm} / \mathrm{Sm}$ are analyzed by liquid scintillation counting over several different beta energy windows, aliquots are also activated, and the resulting ${ }^{155} \mathrm{Sm}$ 's ( $\left.\mathrm{t}_{1 / 2} 22.2 \mathrm{~m}\right) 104 \mathrm{keV}$ gamma are measured to determine the samarium carriers recovery. Pm recoveries (Pm having no stable isotopes) are determined one of two ways. One pathway uses a ${ }^{147} \mathrm{Pm}$ spike that is run with the batch, traced with stable samarium, and a ratio is determined between the Pm and Sm recoveries for the samples. The second pathway is to activate stable $\mathrm{Nd}$, generating ${ }^{149} \mathrm{Pm}\left(\mathrm{t}_{1 / 2} 2.212\right.$ days $)$ from the beta decay of ${ }^{149} \mathrm{Nd}$. The activated $\mathrm{Nd}$ is then added to each sample in conjunction with the Sm carrier, and the ${ }^{151} \mathrm{Sm}$ separations are yielded with the stable Sm carrier, the ${ }^{147} \mathrm{Pm}$ separations are yielded with the ${ }^{149} \mathrm{Pm}$ tracer.

\section{CONCLUSIONS}

The SRNL NAA facility continues to demonstrate the usefulness of low-flux ${ }^{252} \mathrm{Cf}$ sources. Although the facility can not compete with analytical methods such as ICPMS on the basis of sensitivity alone, the facility provides adequate sensitivity for many applications for samples analyzed in bulk. This is a useful advantage on difficult-to-digest matrices. The facility offers invaluable support in the arena of radiochemical separations. Short-lived radio-tracers are generated at the facility which are spiked into samples to serve as chemical yield agents for elements being separated. In cases where suitable radio-tracers can not be generated, the facility 
often helps complete radiometric analyses by providing yields through activation and counting of carriers for elements of interest.

\section{REFERENCES}

1. D. DiPrete, S.F. Peterson, and R.A.Sigg, JRNC, 244, No. 2 (2000) 343.

2. Currie, L.A., Analytical Chemistry 40 No. 3 (1968) 586.

3. Anderson, T.J. ASTM Special Technical Publication, No 698, (1980) 84.3.

4. Bhattacharyya, A.K., and R. Janati. "Safety Issues Related to Disposal of I-129 in a Low Level Radioactive Waste Repository," Proceeding of the Ninth Annual DOE Low Level Radioactive Waste Management Conference, Denver, CO (1987).

5. Environmental Protection Agency, "Prescribed Procedures for Measurement of Radioactivity in Drinking Water,” EPA, EPA-680/4-80-032 (1990) 26.

6. D. P. DiPrete, C. C. DiPrete, and R. A. Sigg Journal of Radioanalytical and Nuclear Chemistry, 263, No 3 (2005) 593 
Figure 1. The Savannah River National Laboratory's ${ }^{252}$ Cf Neutron Activation Analysis Facility.

Figure 2a. Detection limits for individual elements with sensitivities up to 10ppm, irradiating a $5 \mathrm{~g}$ sample for 30 minutes with a $21-\mathrm{mg}{ }^{252} \mathrm{Cf}$ source, decaying for 5 minutes, and counting for 16.6 minutes on a $30 \%$ efficient coaxial germanium detector.

Figure 2b. Detection limits for individual elements with sensitivities from 10ppm to $100 \mathrm{ppm}$, irradiating a $5 \mathrm{~g}$ sample for 30 minutes with a $21-\mathrm{mg}{ }^{252} \mathrm{Cf}$ source, decaying for 5 minutes, and counting for 16.6 minutes on a $30 \%$ efficient coaxial germanium detector.

Figure 2c. Detection limits for individual elements with sensitivities from 100ppm to $500 \mathrm{ppm}$, irradiating a $5 \mathrm{~g}$ sample for 30 minutes with a $21-\mathrm{mg}{ }^{252} \mathrm{Cf}$ source, decaying for 5 minutes, and counting for 16.6 minutes on a $30 \%$ efficient coaxial germanium detector. 


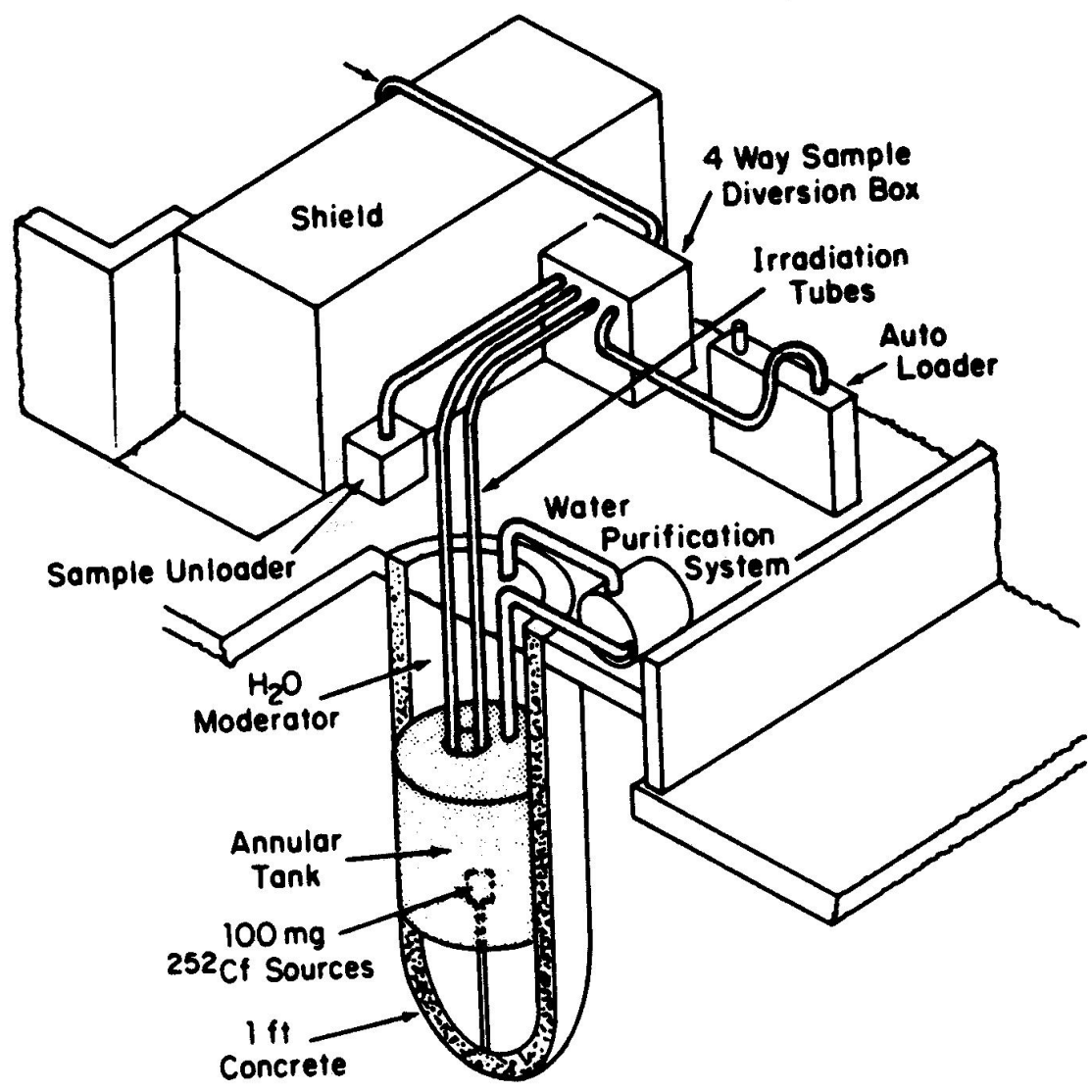




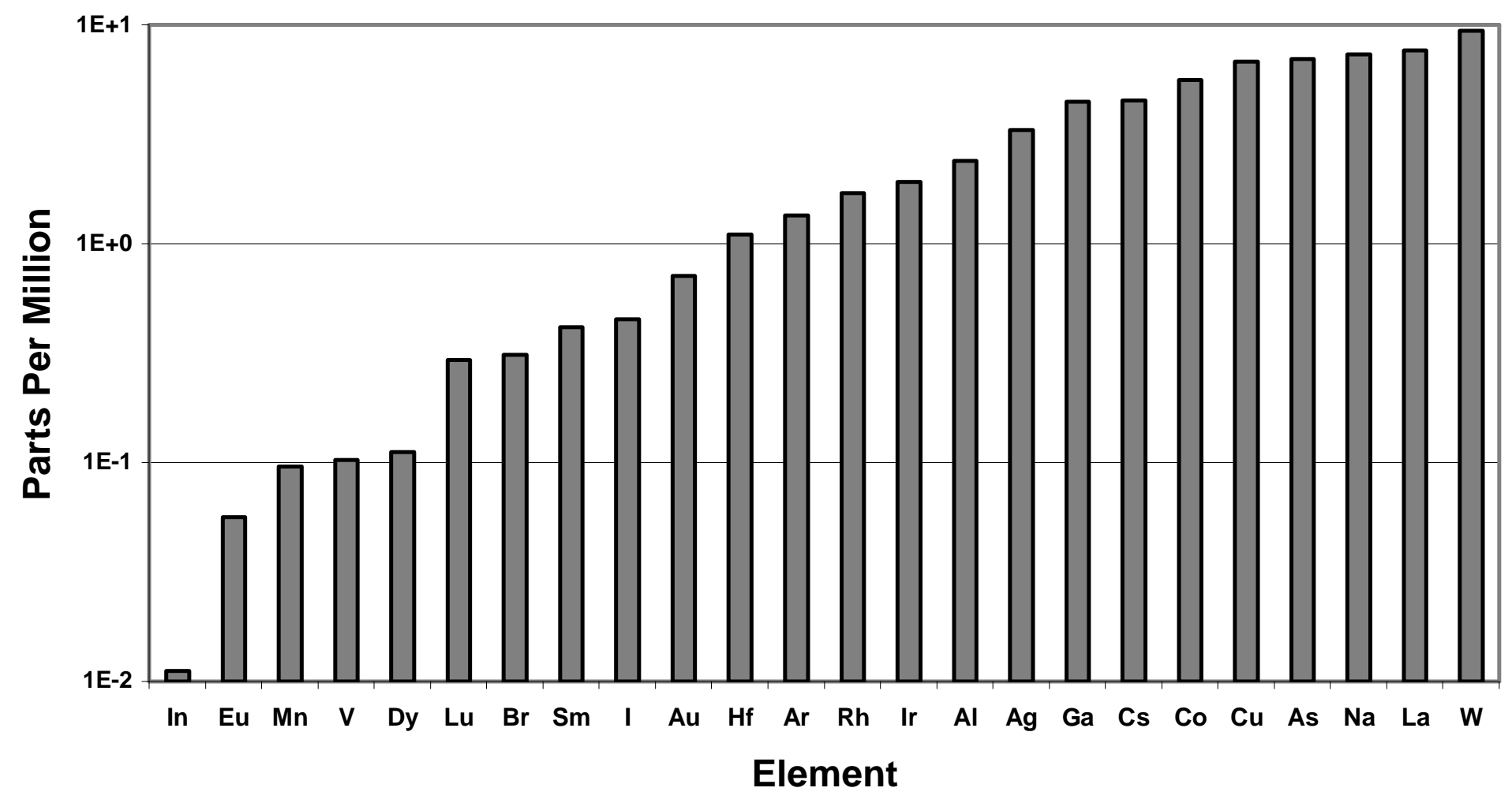




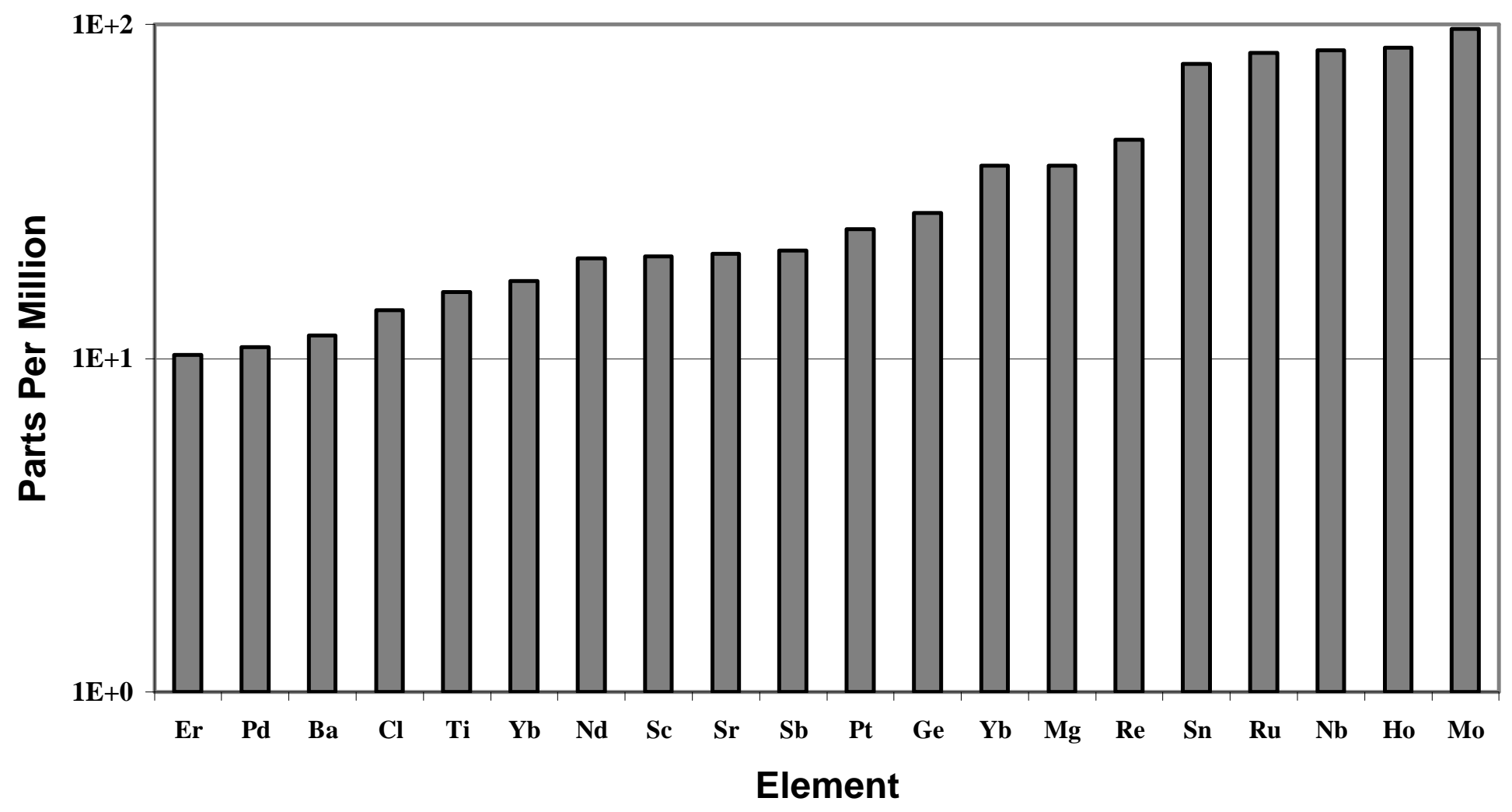




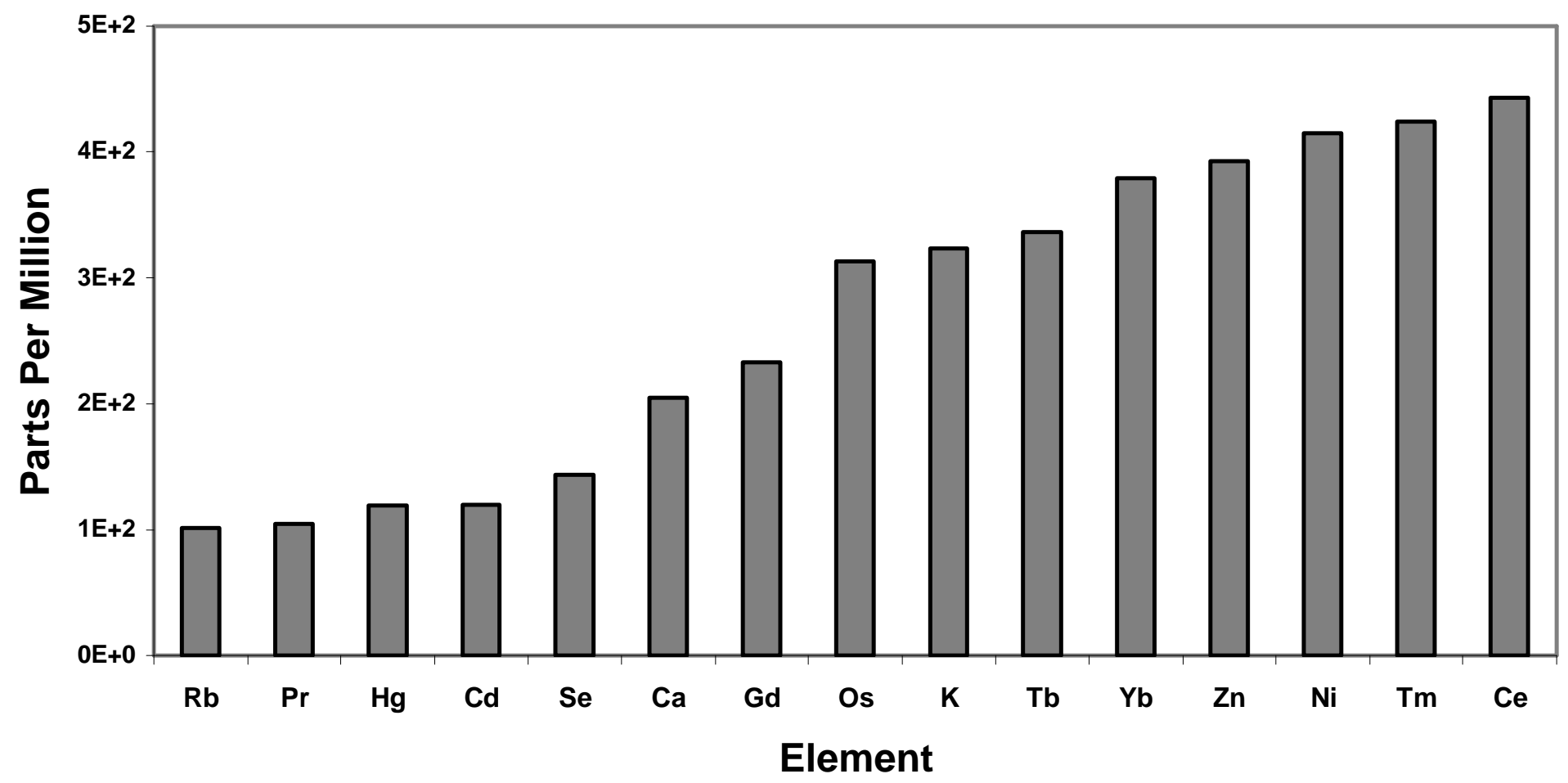

